\title{
Lyapunov Techniques for Stochastic Differential Equations Driven by Fractional Brownian Motion
}

\author{
Caibin Zeng, ${ }^{1,2}$ Qigui Yang, ${ }^{1}$ and YangQuan Chen ${ }^{3}$ \\ ${ }^{1}$ School of Sciences, South China University of Technology, Guangzhou 510640, China \\ ${ }^{2}$ School of Automation Science and Engineering, South China University of Technology, Guangzhou 510640, China \\ ${ }^{3}$ Mechatronics, Embedded Systems and Automation (MESA) Lab, School of Engineering, University of California, Merced, \\ 5200 N Lake Road, Merced, CA 95343, USA
}

Correspondence should be addressed to Caibin Zeng; zeng.cb@mail.scut.edu.cn

Received 2 November 2013; Revised 12 January 2014; Accepted 26 January 2014; Published 11 March 2014

Academic Editor: Weilin Xiao

Copyright (C) 2014 Caibin Zeng et al. This is an open access article distributed under the Creative Commons Attribution License, which permits unrestricted use, distribution, and reproduction in any medium, provided the original work is properly cited.

Little seems to be known about evaluating the stochastic stability of stochastic differential equations (SDEs) driven by fractional Brownian motion ( $\mathrm{fBm}$ ) via stochastic Lyapunov technique. The objective of this paper is to work with stochastic stability criterions for such systems. By defining a new derivative operator and constructing some suitable stochastic Lyapunov function, we establish some sufficient conditions for two types of stability, that is, stability in probability and moment exponential stability of a class of nonlinear SDEs driven by fBm. We will also give an example to illustrate our theory. Specifically, the obtained results open a possible way to stochastic stabilization and destabilization problem associated with nonlinear SDEs driven by fBm.

\section{Introduction}

Fractional Brownian motion ( $\mathrm{fBm}$ ) is a family of Gaussian stochastic processes that appears naturally in the modeling of many situations. Kolmogorov [1] was the first to consider this process and called it "Wiener Spirals." Later, Hurst $[2,3]$ studied the long-term water flow characteristics of the Nile River and the parameter $H$ then got the name "Hurst parameter." Mandelbrot and Van Ness [4] established a stochastic integral representation in terms of a standard Brownian motion. Since the introduction of the above mentioned pioneering work, $\mathrm{fBm}$ has played an increasingly important role in many fields of application such as hydrology, economics, and telecommunications (see [5] for a review).

According to the books [6,7], the standard $\mathrm{fBm}$ $\left(B^{H}(t), t \geq 0\right)$ is defined as a self-similar centered Gaussian process with covariance function

$$
\operatorname{Cov}\left(B^{H}(t), B^{H}(s)\right)=\frac{1}{2}\left(t^{2 H}+s^{2 H}-|t-s|^{2 H}\right),
$$

where Hurst parameter $0<H<1$. When $H=1 / 2$, one recovers of course the usual Brownian motion, so this is a natural one-parameter family of generalizations of the "standard" Brownian motion. When $H \neq 1 / 2$, it was proved in [8] that $\mathrm{fBm}$ is not semimartingale. Therefore, the beautiful classical theory of stochastic analysis [9] is not applicable to stochastic differential equations (SDEs) driven by $\mathrm{fBm}$ with $H \neq 1 / 2$. It is a significant and challenging problem to extend the results in the classical stochastic analysis to these $\mathrm{fBm}$ ones. Over the last years some new techniques have been developed in order to define stochastic integrals with respect to $\mathrm{fBm}[10-21]$. For example, stochastic integral of deterministic functions with respect to $\mathrm{fBm}$ is called Wiener integral, which was defined for the first time in [10]. The stochastic integral of Stratonovich type for $\mathrm{fBm}$ was defined in $[11,12]$. However, the stochastic integral $\int_{0}^{t} f(s) \delta B^{H}(s)$, introduced in $[11,12]$, does not satisfy in general the following property: $E \int_{0}^{t} f(s) \delta B^{H}(s)=0$, which is important in the modeling problem by stochastic differential equations with fractional Gaussian noise as the driving random process. Motivated by this situation, Duncan et al. [13] defined a new stochastic integral of Itô type for $\mathrm{fBm}$ with Hurst parameter 
in the interval $(1 / 2,1)$. This stochastic integral is the limit of Riemann sums defined by means of the Wick products rather than ordinary products. In this paper, we adopt this stochastic integral definition. Then Elliott and Van der Hoek in [16] extended this fractional Itô calculus theory to all Hurst parameter $H \in(0,1)$ and applied it to develop option pricing in a fractional Black-Scholes market. This newly developed theory of stochastic integration with respect to $\mathrm{fBm}$, based on white-noise theory and (Malliavin-type) differentiation, was introduced in [17]. For other definitions of stochastic integrals for $\mathrm{fBm}$ and their relations, we refer to the book [6] for further details.

Recently, some sufficient and necessary conditions for reducing the nonlinear stochastic systems driven by $\mathrm{fBm}$ to the linear ones were constructed in our previous work [22], which provide an effective approach to solve some linear and nonlinear $\mathrm{fBm}$-driven stochastic systems. Indeed, necessary and sufficient conditions were established for stochastic stability of the Black-Scholes model driven by $\mathrm{fBm}$ by means of the Lyapunov exponents and the exact form of the solutions [23]. Unfortunately, it is in general not possible to give explicit expressions for the solutions to SDEs and numerical solution is a cumbersome affair. It is therefore of great interest to study qualitative properties of SDEs driven by $\mathrm{fBm}$ without solving the equations. Therefore, the scope of this paper is to extend the stochastic Lyapunov function technique to SDEs driven by $\mathrm{fBm}$ without solving the considered equations.

We organize this paper as follows. In Section 2, we briefly introduce some necessary notations and stochastic stability concepts associated with SDEs driven by fBm. In Section 3, we state the main results on stability of SDEs driven by $\mathrm{fBm}$ via Lyapunov function technique. In Section 4, we apply the stability criterions to the Ornstein-Uhlenbeck process driven by $\mathrm{fBm}$ by constructing a time-dependent Lyapunov function. The conclusions are drawn in Section 5.

\section{Preliminaries}

2.1. Notations. We consider the $m$-dimensional SDE driven by $\mathrm{fBm}$ of the form

$$
\begin{gathered}
d x(t)=f(x(t), t) d t+g(x(t), t) d B^{H}(t), \\
x\left(t_{0}\right)=x_{0} .
\end{gathered}
$$

We will suppose that (2) satisfies the conditions for a unique global solution as in [7, Section 3.3]. Denote the solution by $x\left(t ; t_{0}, x_{0}\right)$, which has continuous sample paths. Assume furthermore that

$$
f(0, t)=0, \quad g(0, t)=0, \quad \forall t \geq t_{0} .
$$

Then $x(t)=0$ is the trivial solution of (2).

It is convenient herein for us to give a few necessary notations. Let $\mathscr{K}$ denote the family of all continuous nondecreasing functions $\mu: \mathbb{R}_{+} \rightarrow \mathbb{R}_{+}$such that $\mu(0)=0$ and $\mu(r)>0$ if $r>0$. For $h>0$, let $\mathbb{S}_{h}=\left\{x \in \mathbb{R}^{m}:|x|<h\right\}$. A continuous function $V(x, t)$ defined on $\mathbb{S}_{h} \times\left[t_{0}, \infty\right)$ is said to be positive definite (in the sense of Lyapunov) if $V(0, t) \equiv 0$ and, for some $\mu \in \mathscr{K}$,

$$
V(x, t) \geq \mu(|x|), \quad \forall(x, t) \in \mathbb{S}_{h} \times\left[t_{0}, \infty\right) .
$$

A function $V$ is said to be negative definite if $-V$ is positive definite. A continuous nonnegative function $V(x, t)$ is said to be decrescent (i.e., to have an arbitrarily small upper bound) if, for some $\mu \in \mathscr{K}$,

$$
V(x, t) \leq \mu(|x|), \quad \forall(x, t) \in \mathbb{S}_{h} \times\left[t_{0}, \infty\right) .
$$

A function $V(x, t)$ defined on $\mathbb{R}^{n} \times\left[t_{0}, \infty\right)$ is said to be radially unbounded if

$$
\lim _{|x| \rightarrow \infty} \inf _{t \geq t_{0}} V(x, t)=\infty
$$

Let $\mathbb{C}^{2,1}\left(\mathbb{S}_{h} \times\left[t_{0}, \infty\right) ; \mathbb{R}_{+}\right)$denote the family of all nonnegative functions $V(x, t)$ defined on $\mathbb{S}_{h} \times\left[t_{0}, \infty\right)$ such that they are continuously twice differentiable in $x$ and once in $t$.

Define a new derivative operator $L^{H}$ associated with (2) by

$$
\begin{aligned}
L^{H}= & \frac{\partial}{\partial t}+\sum_{i=1}^{m} f_{i}(x(t), t) \frac{\partial}{\partial x_{i}} \\
& +\sum_{i, j=1}^{m} g_{i}(x(t), t) \int_{0}^{t} \phi(v, s) g_{j}(x(v), v) d v \frac{\partial^{2}}{\partial x_{i} \partial x_{j}}
\end{aligned}
$$

where the nonlocal kernel function

$$
\phi(s, t)=H(2 H-1)|s-t|^{2 H-2} .
$$

If $L^{H}$ acts on a function $V \in \mathbb{C}^{2,1}\left(\mathbb{S}_{h} \times\left[t_{0}, \infty\right) ; \mathbb{R}_{+}\right)$, then

$$
\begin{aligned}
L^{H} V(x, t)= & V_{t}(x, t)+V_{x}(x, t) f(x(t), t) \\
& +V_{x x}(x, t) g(x(t), t) \int_{0}^{t} \phi(v, s) g(x(v), v) d v .
\end{aligned}
$$

By fractional Itô formula [13], if $x(t) \in \mathbb{S}_{h}$, then

$$
d V(x, t)=L^{H} V(x, t) d t+V_{x}(x, t) g(x(t), t) d B^{H}(t) .
$$

Remark 1. Compared with the classical Itô (or Stratonovich) SDEs [24], the significant difference is the presence of the nonlocal kernel operator in the new differential operator $L^{H}$.

Remark 2. Since the kernel function $\phi$ is defined only for $1 / 2<H<1$, so the derivative operator $L^{H}$ makes no sense when $0<H<1 / 2$. Thus we assume that $1 / 2<H<1$ throughout this paper.

2.2. Stochastic Stability Concepts. It turns out that there are at least three different types of stochastic stability: stability in probability, moment stability, and almost sure stability. We 
focus on the first two types in this paper since we already studied the third one in our previous paper [23].

We now give the definitions of stability in probability and the $p$ th moment exponential stability, which are the same as those in [24].

Definition 3. The trivial solution of (2) is said to be stochastically stable or stable in probability if, for every pair of $\varepsilon \in$ $(0,1)$ and $r>0$, there exists a $\delta=\delta\left(\varepsilon, r, t_{0}\right)>0$, such that

$$
\mathbb{P}\left\{\left|x\left(t ; t_{0}, x_{0}\right)\right|<r, \forall t \geq t_{0}\right\} \geq 1-\varepsilon,
$$

whenever $\left|x_{0}\right|<\delta$. Otherwise, it is said to be stochastically unstable.

Definition 4. The trivial solution of (2) is said to be stochastically asymptotically stable if it is stochastically stable and, moreover, for every $\varepsilon \in(0,1)$, there exists a $\delta=\delta\left(\varepsilon, t_{0}\right)>0$, such that

$$
\mathbb{P}\left\{\lim _{t \rightarrow \infty} x\left(t ; t_{0}, x_{0}\right)=0\right\} \geq 1-\varepsilon,
$$

whenever $\left|x_{0}\right|<\delta$.

Definition 5. The trivial solution of (2) is said to be stochastically asymptotically stable in the large if it is stochastically stable and, moreover, for $x_{0} \in \mathbb{R}^{m}$

$$
\mathbb{P}\left\{\lim _{t \rightarrow \infty} x\left(t ; t_{0}, x_{0}\right)=0\right\}=1
$$

Definition 6. Assume that $p>0$. The trivial solution of (2) is said to be $p$ th moment exponentially stable if there is a pair of positive constants $\lambda$ and $C$ such that

$$
\mathbb{E}\left[\left|x\left(t ; t_{0}, x_{0}\right)\right|^{p}\right] \leq C\left|x_{0}\right|^{p} e^{-\lambda\left(t-t_{0}\right)}, \quad t \geq t_{0},
$$

for all $x_{0} \in \mathbb{R}^{m}$.

\section{Main Results}

We now extend the stochastic Lyapunov function techniques to the SDEs driven by $\mathrm{fBm}$.

Theorem 7. If there exists a positive-definite function $V(x, t) \in$ $\mathbb{C}^{2,1}\left(\mathbb{S}_{h} \times\left[t_{0}, \infty\right) ; \mathbb{R}_{+}\right)$such that

$$
L^{H} V(x, t) \leq 0
$$

for all $(x, t) \in \mathbb{S}_{h} \times\left[t_{0}, \infty\right)$, then the trivial solution of (2) is stochastically stable.

Proof. By the definition of a positive-definite function, we know that $V(0, t) \equiv 0$, and there is a function $\mu \in \mathscr{K}$, such that

$$
V(x, t) \geq \mu(|x|), \quad \forall(x, t) \in \mathbb{S}_{h} \times\left[t_{0}, \infty\right) .
$$

Let $\varepsilon \in(0,1)$ and $r>0$ be arbitrary. Without loss of generality, we assume that $r<h$.
Indeed, by the continuity of $V(x, t)$ and the fact that $V\left(0, t_{0}\right)=0$, we can find a $\delta=\delta\left(\varepsilon, r, t_{0}\right)>0$, such that

$$
\left.\frac{1}{\varepsilon_{x \in \mathbb{S}_{\delta}}} \sup _{x, t_{0}}\right) \leq \mu(r) .
$$

It is not difficult to see that $\delta<r$. Now fix the initial value $x_{0} \in \mathbb{S}_{\delta}$ arbitrarily and write $x\left(t ; t_{0}, x_{0}\right)$ as $x(t)$ for simplicity.

Let $\tau$ be the first exit time of $x(t)$ from $\mathbb{S}_{r}$; that is,

$$
\tau=\inf \left\{t \geq t_{0}: x(t) \notin \mathbb{S}_{r}\right\} \text {. }
$$

For $t \geq t_{0}$, it follows that

$$
\begin{aligned}
V(x(\tau \wedge t), \tau \wedge t)= & V\left(x_{0}, t_{0}\right)+\int_{t_{0}}^{\tau \wedge t} L^{H} V(x(s), s) d s \\
& +\int_{t_{0}}^{\tau \wedge t} V_{x}(x(s), s) g(x(s), s) d B^{H}(s) .
\end{aligned}
$$

Taking the expectation on both sides and utilizing the condition $L^{H} V(x, t) \leq 0$, we have

$$
\mathbb{E}[V(x(\tau \wedge t), \tau \wedge t)] \leq V\left(x_{0}, t_{0}\right) .
$$

Note that

$$
|x(\tau \wedge t)|=|x(\tau)|=r,
$$

if $\tau \leq t$. Hence, by (16), we further get

$$
\begin{aligned}
\mathbb{E}[V(x(\tau \wedge t), \tau \wedge t)] & \geq \mathbb{E}\left[I_{\{\tau \leq t\}} V(x(\tau), \tau)\right] \\
& \geq \mu(r) \mathbb{P}\{\tau \leq t\} .
\end{aligned}
$$

Together with (17), (20), and (22), we have

$$
\mathbb{P}\{\tau \leq t\} \leq \varepsilon .
$$

Letting $t \rightarrow \infty$, we get

$$
\mathbb{P}\{\tau<\infty\} \leq \varepsilon
$$

That is,

$$
\begin{aligned}
\mathbb{P}\left\{|x(t)|<r, \forall t \geq t_{0}\right\} & =\mathbb{P}\{\tau=\infty\} \\
& =1-\mathbb{P}\{\tau<\infty\} \geq 1-\varepsilon .
\end{aligned}
$$

Thus the proof is established.

Theorem 8. If there is a positive-definite, decrescent function $V(x, t) \in \mathbb{C}^{2,1}\left(\mathbb{S}_{h} \times\left[t_{0}, \infty\right) ; \mathbb{R}_{+}\right)$such that $L^{H} V(x, t)$ is negative definite, then the trivial solution of (2) is stochastically asymptotically stable.

Proof. By the condition that $L^{H} V(x, t)$ is negative definite, there exists a function $\mu \in \mathscr{K}$, such that

$$
-L^{H} V(x, t) \geq \mu(|x|), \quad \forall(x, t) \in \mathbb{S}_{h} \times\left[t_{0}, \infty\right) .
$$


Then it follows from the definition of $\mathscr{K}$-class functional that

$$
L^{H} V(x, t) \leq 0 \text {. }
$$

This, together with the positive-definite property of function $V(x, t)$, satisfies the required conditions in Theorem 7 . It means that the trivial solution is stochastically stable. So we only need to show that, for any $\varepsilon \in(0,1)$, there is a $\delta=$ $\delta\left(\varepsilon, t_{0}\right)>0$ such that

$$
\mathbb{P}\left\{\lim _{t \rightarrow \infty} x\left(t ; t_{0}, x_{0}\right)=0\right\} \geq 1-\varepsilon,
$$

whenever $\left|x_{0}\right|<\delta$. Note that the assumptions on function $V(x, t)$ mean that $V(0, t) \equiv 0$, and moreover, there are three functions $\mu_{1}, \mu_{2}, \mu_{3} \in \mathscr{K}$ such that

$$
\mu_{1}(|x|) \leq V(x, t) \leq \mu_{2}(x, t), \quad L^{H} V(x, t) \leq-\mu_{3}(|x|)
$$

for all $(x, t) \in \mathbb{S}_{h} \times\left[t_{0}, \infty\right)$.

Let $\varepsilon \in(0,1)$ be arbitrary, and by Theorem 7 , there is a $\delta=\delta\left(\varepsilon, t_{0}\right)>0$, such that

$$
\mathbb{P}\left\{\left|x\left(t ; t_{0}, x_{0}\right)\right|<\frac{h}{2}\right\} \geq 1-\frac{\varepsilon}{4}, \quad \forall x_{0} \in \mathbb{S}_{\delta} .
$$

For any $x_{0} \in \mathbb{S}_{\delta}$, write $x\left(t ; t_{0}, x_{0}\right)=x(t)$ simply. Let $0<\beta<$ $\left|x_{0}\right|$ be arbitrary and choose $0<\alpha<\beta$ sufficiently small for

$$
\frac{\mu_{2}(\alpha)}{\mu_{1}(\beta)} \leq \frac{\varepsilon}{4}
$$

Define the stopping times

$$
\begin{gathered}
\tau_{\alpha}=\inf \left\{t \geq t_{0}:|x(t)| \leq \alpha\right\}, \\
\tau_{h}=\inf \left\{t \geq t_{0}:|x(t)| \geq \frac{h}{2}\right\} .
\end{gathered}
$$

For any $t \geq t_{0}$, it follows from (29) that

$$
\begin{aligned}
0 & \leq \mathbb{E}\left[V\left(x\left(\tau_{\alpha} \wedge \tau_{h} \wedge t\right), \tau_{\alpha} \wedge \tau_{h} \wedge t\right)\right] \\
& =V\left(x_{0}, t_{0}\right)+\mathbb{E}\left[\int_{t_{0}}^{\tau_{\alpha} \wedge \tau_{h} \wedge t} L^{H} V(x(s), s) d s\right] \\
& \leq V\left(x_{0}, t_{0}\right)-\mu_{3}(\alpha) \mathbb{E}\left[\tau_{\alpha} \wedge \tau_{h} \wedge\left(t-t_{0}\right)\right] .
\end{aligned}
$$

On the other hand, it yields

$$
\begin{aligned}
\mathbb{E}\left[\tau_{\alpha} \wedge\right. & \left.\tau_{h} \wedge\left(t-t_{0}\right)\right] \\
= & \mathbb{E}\left[\tau_{\alpha} \wedge \tau_{h} \wedge\left(t-t_{0}\right)\right] I_{\left\{\tau_{\alpha} \wedge \tau_{h} \geq t\right\}} \\
& +\left(\tau_{\alpha} \wedge \tau_{h} \wedge\left(t-t_{0}\right)\right) I_{\left\{\tau_{\alpha} \wedge \tau_{h}<t\right\}} \\
\geq & \mathbb{E}\left[\tau_{\alpha} \wedge \tau_{h} \wedge\left(t-t_{0}\right)\right] I_{\left\{\tau_{\alpha} \wedge \tau_{h} \geq t\right\}} \\
= & \left(t-t_{0}\right) \mathbb{P}\left\{\tau_{\alpha} \wedge \tau_{h} \geq t\right\} .
\end{aligned}
$$

It then follows from (33) and (34) that

$$
\left(t-t_{0}\right) \mathbb{P}\left\{\tau_{\alpha} \wedge \tau_{h} \geq t\right\} \leq \mathbb{E}\left[\tau_{\alpha} \wedge \tau_{h} \wedge\left(t-t_{0}\right)\right] \leq \frac{V\left(x_{0}, t_{0}\right)}{\mu_{3}(\alpha)} .
$$

Letting $t \rightarrow \infty$, we have

$$
\mathbb{P}\left\{\tau_{\alpha} \wedge \tau_{h}<\infty\right\}=1 .
$$

On the other hand, it follows from (30) and (32) that

$$
\mathbb{P}\left\{\tau_{h}<\infty\right\} \leq \frac{\varepsilon}{4} .
$$

Hence,

$$
\begin{aligned}
1 & =\mathbb{P}\left\{\tau_{\alpha} \wedge \tau_{h}<\infty\right\} \leq \mathbb{P}\left\{\tau_{\alpha}<\infty\right\}+\mathbb{P}\left\{\tau_{h}<\infty\right\} \\
& \leq \mathbb{P}\left\{\tau_{\alpha}<\infty\right\}+\frac{\varepsilon}{4},
\end{aligned}
$$

which yields

$$
\mathbb{P}\left\{\tau_{\alpha}<\infty\right\} \geq 1-\frac{\varepsilon}{4} .
$$

Choose $\theta$ sufficiently large for

$$
\mathbb{P}\left\{\tau_{\alpha}<\theta\right\} \geq 1-\frac{\varepsilon}{2} .
$$

Then

$$
\begin{aligned}
\mathbb{P}\left\{\tau_{\alpha}<\tau_{h} \wedge \theta\right\} & \geq \mathbb{P}\left\{\left\{\tau_{\alpha}<\theta\right\} \cap\left\{\tau_{h}=\infty\right\}\right\} \\
& \geq \mathbb{P}\left\{\tau_{\alpha}<\theta\right\}-\mathbb{P}\left\{\tau_{h}<\infty\right\} \geq 1-\frac{3 \varepsilon}{4}
\end{aligned}
$$

Now, define two stopping times

$$
\begin{gathered}
\sigma= \begin{cases}\tau_{\alpha}, & \text { if } \tau_{\alpha}<\tau_{h} \wedge \theta, \\
\infty, & \text { otherwise, }\end{cases} \\
\tau_{\beta}=\inf \{t>\sigma:|x(t)| \geq \beta\} .
\end{gathered}
$$

For any $t \geq \theta$, it follows

$$
\mathbb{E}\left[V\left(x\left(\tau_{\beta} \wedge t\right), \tau_{\beta} \wedge t\right)\right] \leq \mathbb{E}[V(x(\sigma \wedge t), \sigma \wedge t)] .
$$

Noting that

$$
V\left(x\left(\tau_{\beta} \wedge t\right), \tau_{\beta} \wedge t\right)=V(x(\sigma \wedge t), \sigma \wedge t)=V(x(t), t)
$$

on $\omega \in\left\{\tau_{\alpha} \geq \tau_{h} \wedge \theta\right\}$, we get

$$
\begin{array}{r}
\mathbb{E}\left[I_{\left\{\tau_{\alpha}<\tau_{h} \wedge \theta\right\}} V\left(x\left(\tau_{\beta} \wedge t\right), \tau_{\beta} \wedge t\right)\right] \\
\leq \mathbb{E}\left[I_{\left\{\tau_{\alpha}<\tau_{h} \wedge \theta\right\}} V\left(x\left(\tau_{\alpha}\right), \tau_{\alpha}\right)\right] .
\end{array}
$$

Utilizing (30) and the fact that $\left\{\tau_{\beta} \leq t\right\} \subset\left\{\tau_{\alpha}<\tau_{h} \wedge \theta\right\}$ we further have

$$
\mu_{1}(\beta) \mathbb{P}\left\{\tau_{\beta} \leq t\right\} \leq \mu_{2}(\alpha)
$$


Together with (31), we deduce that

$$
\mathbb{P}\left\{\tau_{\beta} \leq t\right\} \leq \frac{\varepsilon}{4} .
$$

Letting $t \rightarrow \infty$, we have

$$
\mathbb{P}\left\{\tau_{\beta} \leq \infty\right\} \leq \frac{\varepsilon}{4} .
$$

It follows from (41) that

$$
\begin{aligned}
\mathbb{P}\{\sigma & \left.<\infty, \tau_{\beta}=\infty\right\} \\
& \geq \mathbb{P}\left\{\tau_{\alpha}<\tau_{h} \wedge \theta\right\}-\mathbb{P}\left\{\tau_{\beta}<\infty\right\} \geq 1-\varepsilon
\end{aligned}
$$

This implies that

$$
\mathbb{P}\left\{\omega: \lim _{t \rightarrow \infty} \sup |x(t)| \leq \beta\right\} \geq 1-\varepsilon .
$$

Since $\beta$ is arbitrary, we must have

$$
\mathbb{P}\left\{\omega: \lim _{t \rightarrow \infty} \sup |x(t)|=0\right\} \geq 1-\varepsilon,
$$

as required. The proof is complete.

Theorem 9. If there is a positive-definite, decrescent, radially unbounded function $V(x, t) \in \mathbb{C}^{2,1}\left(\mathbb{S}_{h} \times\left[t_{0}, \infty\right) ; \mathbb{R}_{+}\right)$, such that $L^{H} V(x, t)$ is negative definite, then the trivial solution of equation is stochastically asymptotically stable in the large.

Proof. By the proof of Theorem 8, the trivial solution of equation is stochastically stable. So we only need to show that

$$
\mathbb{P}\left\{\lim _{t \rightarrow \infty} x\left(t ; t_{0}, x_{0}\right)=0\right\}=1,
$$

for all $x_{0} \in \mathbb{R}^{m}$; fix any $x_{0}$ and write $x\left(t ; t_{0}, x_{0}\right)=x(t)$ again. Let $\varepsilon \in(0,1)$ be arbitrary; since $V(x, t)$ is radially unbounded, we can find an $h>\left|x_{0}\right|$ sufficiently large for

$$
\lim _{|x| \geq h, t \geq t_{0}} V(x, t) \geq \frac{4 V\left(x_{0}, t_{0}\right)}{\varepsilon} .
$$

Define the stopping time

$$
\tau_{h}=\inf \left\{t \geq t_{0}:|x(t)| \geq h\right\} .
$$

For any $t \geq t_{0}$, it follows that

$$
\mathbb{E}\left[V\left(x\left(\tau_{h} \wedge t\right), \tau_{h} \wedge t\right)\right] \leq V\left(x_{0}, t_{0}\right) .
$$

But, it follows from (53) that

$$
\mathbb{E}\left[V\left(x\left(\tau_{h} \wedge t\right), \tau_{h} \wedge t\right)\right] \geq \frac{4 V\left(x_{0}, t_{0}\right)}{\varepsilon} \mathbb{P}\left\{\tau_{h} \leq t\right\} .
$$

It then follows from (55) that

$$
\mathbb{P}\left\{\tau_{h} \leq t\right\} \leq \frac{\varepsilon}{4}
$$

Let $t \rightarrow \infty$; we have

$$
\mathbb{P}\left\{\tau_{h}<\infty\right\} \leq \frac{\varepsilon}{4} .
$$

That is,

$$
\mathbb{P}\left\{|x(t)|<h, \forall t \geq t_{0}\right\} \geq 1-\frac{\varepsilon}{4} .
$$

From here, we can show in the same way as the proof of Theorem 8 that

$$
\mathbb{P}\left\{\lim _{t \rightarrow \infty} x(t)=0\right\} \geq 1-\varepsilon .
$$

Since $\varepsilon$ is arbitrary, the required equation (52) must hold and thus the proof is complete.

Next we focus on the $p$ th moment exponential stability of (2) and always let $p>0$. Now we establish a sufficient criterion for the $p$ th moment exponential stability by using a stochastic Lyapunov function.

Theorem 10. Assume that there exist a function $V(x, t) \in$ $\mathbb{C}^{2,1}\left(\mathbb{S}_{h} \times\left[t_{0}, \infty\right) ; \mathbb{R}_{+}\right)$and positive constants $c_{1}, c_{2}, c_{3}$, such that

$$
\begin{aligned}
& \text { (i) } c_{1}|x|^{p} \leq V(x, t) \leq c_{2}|x|^{p}, \\
& \text { (ii) } L^{H} V(x, t) \leq-c_{3} V(x, t),
\end{aligned}
$$

for all $(x, t)$; then

$$
\mathbb{E}\left[\left|x\left(t ; t_{0}, x_{0}\right)\right|^{p}\right] \leq \frac{c_{2}}{c_{1}}\left|x_{0}\right|^{p} e^{-c_{3}\left(t-t_{0}\right)},
$$

for all $x_{0} \in R^{m}$ and $t \geq t_{0}$. In other words, the trivial solution of (2) is pth moment exponentially stable and pth moment Lyapunov exponent should not be greater than $-c_{3}$.

Proof. Fix any $x_{0} \in \mathbb{R}^{m}$ and write $x\left(t ; t_{0}, x_{0}\right)=x(t)$. For each $n \geq\left|x_{0}\right|$, we define the stopping time

$$
\tau_{n}=\inf \left\{t \geq t_{0}:|x(t)| \geq n\right\} .
$$

Clearly, $\tau_{n} \rightarrow \infty$ as $n \rightarrow \infty$ almost surely.

By fractional Itô formula, we can derive that, for $t \geq t_{0}$,

$$
e^{\mathcal{C}_{3}\left(t \wedge \tau_{n}-t_{0}\right)} V\left(\left(t \wedge \tau_{n}\right), t \wedge \tau_{n}\right)
$$

$$
=V\left(x_{0}, t_{0}\right)+c_{3} \int_{0}^{t \wedge \tau_{n}} e^{\mathcal{c}_{3}\left(s-t_{0}\right)} V(x(s), s) d s
$$$$
+\int_{0}^{t \wedge \tau_{n}} e^{\mathcal{C}_{3}\left(s-t_{0}\right)} V_{t}(x(s), s) d s
$$$$
+\int_{0}^{t \wedge \tau_{n}} e^{\mathcal{C}_{\mathcal{3}}\left(s-t_{0}\right)} V_{x x}(x(s), s) g(x(s), s)
$$

$$
\times \int_{0}^{t} \phi(v, s) g(x(v), v) d v d s
$$

$$
\begin{aligned}
& +\int_{0}^{t \wedge \tau_{n}} e^{\mathcal{c}_{3}\left(s-t_{0}\right)} V_{x}(x(s), s) f(x(s), s) d s \\
& +\int_{0}^{t \wedge \tau_{n}} e^{\mathcal{c}_{3}\left(s-t_{0}\right)} V_{x}(x(s), s) g(x(s), s) d s .
\end{aligned}
$$


Taking expectation on both sides of (64) and using (9) give

$$
\begin{aligned}
& \mathbb{E}\left[e^{c_{3}\left(t \wedge \tau_{n}-t_{0}\right)} V\left(\left(t \wedge \tau_{n}\right), t \wedge \tau_{n}\right)\right] \\
& =V\left(x_{0}, t_{0}\right) \\
& \quad+\mathbb{E}\left[\int_{0}^{t \wedge \tau_{n}} e^{c_{3}\left(s-t_{0}\right)}\left(c_{3} V(x(s), s)+L^{H}(x(s), s)\right) d s\right] .
\end{aligned}
$$

Using condition (ii) in (61), we then obtain that

$$
\mathbb{E}\left[e^{c_{3}\left(t \wedge \tau_{n}-t_{0}\right)} V\left(\left(t \wedge \tau_{n}\right), t \wedge \tau_{n}\right)\right] \leq V\left(x_{0}, t_{0}\right) .
$$

Combining (66) and condition (i) in (61) gives that

$$
c_{1} e^{c_{3}\left(t \wedge \tau_{n}-t_{0}\right)} \mathbb{E}\left[\left|x\left(t \wedge \tau_{n}\right)\right|^{p}\right] \leq V\left(x_{0}, t_{0}\right) \leq c_{2}\left|x_{0}\right|^{p} .
$$

Letting $n \rightarrow \infty$ yields that

$$
c_{1} e^{c_{3}\left(t-t_{0}\right)} \mathbb{E}\left[|x(t)|^{p}\right] \leq c_{2}\left|x_{0}\right|^{p},
$$

which implies

$$
\mathbb{E}\left[|x(t)|^{p}\right] \leq \frac{c_{2}}{c_{1}}\left|x_{0}\right|^{p} e^{-c_{3}\left(t-t_{0}\right)}
$$

as desired. Therefore the proof is complete.

Remark 11. Note that Hölder inequality implies

$$
\left(\mathbb{E}\left[|x|^{r}\right]\right)^{1 / r} \leq\left(\mathbb{E}\left[|x|^{p}\right]\right)^{1 / p}
$$

for $0<r<p$. Then $p$ th moment exponential stability implies $r$ th moment exponential stability. In particular, when $p=2$, it is usually said to be exponentially stable in square-mean sense.

Remark 12. It should also be pointed out that when $H=$ $1 / 2$, the statements in these theorems reduce to Itô SDEs [24]; when $g(x, t)=0$, these statements reduce to the corresponding deterministic ones.

Remark 13. It would be very hard to study the stochastic stability of some SDEs driven by $\mathrm{fBm}$ via Lyapunov function approach because of the nonlocal property of the kernel function $\phi(\cdot, \cdot)$.

Remark 14. The greatest disadvantage of the stochastic Lyapunov technique is that no universal method has been given which enables you to find a Lyapunov function or determine that no such function exists.

\section{Two Examples}

Through the above discussion, we have established some stochastic-Lyapunov-function-based stability criterions for the SDEs (2) driven by fBm. Now we apply the obtained criterions to check the stochastic stability of Ornstein-Uhlenbeck process driven by $\mathrm{fBm}$ and nonlinear radial OrnsteinUhlenbeck driven by $\mathrm{fBm}$. To do this, the procedure is to construct some suitable stochastic Lyapunov function and verify the required properties of a new derivative operator $L^{H}$.
4.1. Example 1. Precisely, the Ornstein-Uhlenbeck process reads [25]

$$
d x(t)=-\theta x(t) d t+\sigma d B^{H}(t)
$$

where $B^{H}(t)$ is a standard $\mathrm{fBm}$ with Hurst parameter $1 / 2<$ $H<1$ and $\theta>0, \sigma$ are arbitrary constants. It is obvious that (71) satisfies the conditions for a unique global solution as in [7].

Proposition 15. Equation (71) is stochastically stable.

Proof. We construct the time-dependent stochastic Lyanpunov function

$$
V(x, t)=\exp (-\lambda \theta t) x^{4}
$$

for $\lambda>0$ sufficiently large.

First we check the positive-definite property of function (72). It is easy to observe that there are positive constants $m$ and $M$ such that

$$
m \leq \exp (-\lambda \theta t) \leq M
$$

Obviously, $V(0, t)=0$ and there exists $\mathscr{K}$-class function

$$
\mu_{1}(x)=m x^{4}
$$

such that

$$
V(x, t) \geq \mu_{1}(|x|) .
$$

Then we check the nonnegative property of the operator $L^{H}$. In fact, it follows from (9) that

$$
\begin{aligned}
L^{H} V(x, t)= & V_{t}(x, t)+V_{x}(x, t)(-\theta x) \\
& +\sigma^{2} V_{x x}(x, t) \int_{0}^{t} \phi(v, t) d v \\
= & -\lambda \theta \exp (-\lambda \theta t) x^{4}-4 \theta \exp (-\lambda \theta t) x^{4} \\
& +12 H \sigma^{2} t^{2 H-1} \exp (-\lambda \theta t) x^{2} \\
= & -(\lambda+4) \theta \exp (-\lambda \theta t) x^{4} \\
& +12 H \sigma^{2} t^{2 H-1} \exp (-\lambda \theta t) x^{2} .
\end{aligned}
$$

Note the first term in the right hand side of (76) and choose $\lambda$ sufficiently large such that the second term vanishes. Thus,

$$
L^{H} V(x, t) \leq 0
$$

Therefore, we prove this proposition according to Theorem 7.

Proposition 16. Equation (71) is stochastically asymptotically stable.

Proof. We construct the same stochastic Lyapunov function

$$
V(x, t)=\exp (-\lambda \theta t) x^{4}
$$


By the proof of Proposition 15, we know that function (72) is positive definite.

Now we verify the decrescent property of (72). In fact, it is easy to observe that $V(x, t) \geq 0$, and there exists a $\mathscr{K}$-class functional

$$
\mu_{2}(x)=M x^{4},
$$

such that

$$
V(x, t) \leq \mu_{2}(|x|) .
$$

Next we only need to check the nonnegative property of operator $L^{H}$, that is, to check that $-L^{H} V(x, t)$ is positive definite. In fact, it is easy to see that $-L^{H} V(0, t)=0$, and there exists $\mathscr{K}$-class functional

$$
\begin{aligned}
\mu_{3}(x)= & \left(\lambda+4-\varepsilon_{1}\right) \theta \exp (-\lambda \theta t) x^{4} \\
& -12 H \sigma^{2} t^{2 H-1} \exp (-\lambda \theta t) x^{2}
\end{aligned}
$$

such that

$$
-L^{H} V(x, t) \geq \mu_{3}(|x|),
$$

where $\varepsilon_{1}>0$ is arbitrary.

Therefore, we prove this proposition according to Theorem 8

Proposition 17. Equation (71) is stochastically asymptotically stable in the large.

Proof. We construct the same stochastic Lyapunov function

$$
V(x, t)=\exp (-\lambda \theta t) x^{4}
$$

By the proofs of Propositions 15 and 16, we know that function (72) is positive definite and decrescent, and the derivative operator $L^{H}$ is negative definite.

So we only need to check that function (72) is radially unbounded. In fact, it follows from the definition of radially unbounded that

$$
\lim _{|x| \rightarrow \infty} \inf _{t \geq 0}(x, t)=\lim _{|x| \rightarrow \infty} m x^{4}=\infty .
$$

Therefore, we prove this proposition according to Theorem 9 .

Proposition 18. Assume that $p>0$. Equation (71) is $p$ th moment exponentially stable and the pth moment Lyapunov exponent should not be greater than $-\left(\lambda+4-\varepsilon_{2}\right) \theta$, where $\varepsilon_{2}>0$ is an arbitrary constant.

Proof. We first consider $p \geq 4$. Let $p=2(q+1)$, where $q \geq 1$. We consider the stochastic Lyapunov function

$$
V(x, t)=\exp (-\lambda \theta t) x^{p}
$$

Choose $c_{1}=m$ and $c_{2}=M$; then

$$
c_{1} x^{p} \leq V(x, t) \leq c_{2} x^{p}
$$

which implies that the condition (i) in (61) is satisfied.

On the other hand, by (76) there exists $\varepsilon_{2}>0$ such that

$$
\begin{aligned}
L^{H} V(x, t)= & -(\lambda+4) \theta \exp (-\lambda \theta t) x^{p} \\
& +12 H \sigma^{2} t^{2 H-1} \exp (-\lambda \theta t) x^{2 q} \\
\leq & -\left(\lambda+4-\varepsilon_{2}\right) \theta \exp (-\lambda \theta t) x^{p} \\
= & -\left(\lambda+4-\varepsilon_{2}\right) \theta V(x, t) .
\end{aligned}
$$

Let $c_{3}=\left(\lambda+4-\varepsilon_{2}\right) \theta$; then condition (ii) in (61) is satisfied.

According to Theorem 10, (71) is pth moment exponentially stable, where $p \geq 4$.

When $0<p<4$, by Remark 11, (71) is also $p$ th moment exponentially stable.

Therefore, (71) is also $p$ th moment exponentially stable for all $p>0$, and the $p$ th moment Lyapunov exponent should not be greater than $-\left(\lambda+1-\varepsilon_{2}\right) \theta$.

4.2. Example 2. The nonlinear Ornstein-Uhlenbeck model driven by an $\mathrm{fBm}$ is given by

$$
d X(t)=\left(-\theta X^{-1}(t)-X(t)\right)+\sigma B^{H}(t),
$$

where $B^{H}(t)$ is a standard $\mathrm{fBm}$ with Hurst parameter $1 / 2<$ $H<1$ and $\theta>0, \sigma$ are arbitrary constants.

Proposition 19. Equation (88) is stochastically stable.

Proof. We construct the time-dependent stochastic Lyapunov function

$$
V(x, t)=\exp (-\lambda t) x^{4},
$$

for $\lambda>0$ sufficiently large.

First we check the positive-definite property of function (89). It is easy to observe that there are positive constants $m$ and $M$ such that

$$
m \leq \exp (-\lambda t) \leq M .
$$

Obviously, $V(0, t)=0$ and there exists $\mathscr{K}$-class function

$$
\mu_{1}(x)=m x^{4},
$$

such that

$$
V(x, t) \geq \mu_{1}(|x|) .
$$

Then we check the nonnegative property of the operator $L^{H}$. In fact, it follows from (9) that

$$
\begin{aligned}
L^{H} V(x, t)= & V_{t}(x, t)+V_{x}(x, t)\left(-\theta x^{-1}(t)-x(t)\right) \\
& +\sigma^{2} V_{x x}(x, t) \int_{0}^{t} \phi(\nu, t) d v \\
= & -\lambda \exp (-\lambda t) x^{4}-4 \theta \exp (-\lambda t) x^{2}-4 \exp (-\lambda t) x^{4} \\
& +12 H \sigma^{2} t^{2 H-1} \exp (-\lambda t) x^{2} \\
= & -(\lambda+4) \exp (-\lambda \theta t) x^{4}-4 \theta \exp (-\lambda t) x^{2} \\
& +12 H \sigma^{2} t^{2 H-1} \exp (-\lambda t) x^{2} .
\end{aligned}
$$


Note the first two terms in the right hand side of (93) and choose $\lambda$ sufficiently large such that the third term vanishes. Thus,

$$
L^{H} V(x, t) \leq 0
$$

Therefore, we prove this proposition according to Theorem 7.

Proposition 20. Equation (88) is stochastically asymptotically stable.

Proof. We construct the same stochastic Lyapunov function

$$
V(x, t)=\exp (-\lambda t) x^{4} .
$$

By the proof of Proposition 19, we know that function (89) is positive definite.

Now we verify the decrescent property of (89). In fact, it is easy to observe that $V(x, t) \geq 0$, and there exists a $\mathscr{K}$-class functional

$$
\mu_{2}(x)=M x^{4}
$$

such that

$$
V(x, t) \leq \mu_{2}(|x|) .
$$

Next we only need to check the nonnegative property of operator $L^{H}$, that is, to check that $-L^{H} V(x, t)$ is positive definite. In fact, it is easy to see that $-L^{H} V(0, t)=0$, and there exists $\mathscr{K}$-class functional

$$
\begin{aligned}
\mu_{3}(x)= & \left(\lambda+4-\varepsilon_{3}\right) \exp (-\lambda \theta t) x^{4} \\
& +4 \theta \exp (-\lambda t) x^{2}-12 H \sigma^{2} t^{2 H-1} \exp (-\lambda t) x^{2},
\end{aligned}
$$

such that

$$
-L^{H} V(x, t) \geq \mu_{3}(|x|),
$$

where $\varepsilon_{1}>0$ is arbitrary.

Therefore, we prove this proposition according to Theorem 8 .

Proposition 21. Assume that $p>0$. Equation (88) is $p$ th moment exponentially stable and the pth moment Lyapunov exponent should not be greater than $-\left(\lambda+4-\varepsilon_{4}\right)$, where $\varepsilon_{4}>0$ is an arbitrary constant.

Proof. We first consider $p \geq 4$. Let $p=2(q+1)$, where $q \geq 1$. We consider the stochastic Lyapunov function

$$
V(x, t)=\exp (-\lambda \theta t) x^{p}
$$

Choose $c_{1}=m$ and $c_{2}=M$; then

$$
c_{1} x^{p} \leq V(x, t) \leq c_{2} x^{p}
$$

which implies that the condition (i) in (61) is satisfied.
On the other hand, by (93) there exists $\varepsilon_{2}>0$ such that

$$
\begin{aligned}
L^{H} V(x, t)= & -(\lambda+4) \exp (-\lambda \theta t) x^{p}-4 \theta \exp (-\lambda t) x^{2 q} \\
& +12 H \sigma^{2} t^{2 H-1} \exp (-\lambda t) x^{2 q} \\
\leq & -\left(\lambda+4-\varepsilon_{4}\right) \exp (-\lambda \theta t) x^{p} \\
= & -\left(\lambda+4-\varepsilon_{2}\right) V(x, t) .
\end{aligned}
$$

Let $c_{3}=\left(\lambda+4-\varepsilon_{4}\right)$, and then condition (ii) in (61) is satisfied. According to Theorem 10, (88) is pth moment exponentially stable, where $p \geq 4$.

When $0<p<4$, by Remark 11, (88) is also $p$ th moment exponentially stable.

Therefore, (88) is also $p$ th moment exponentially stable for all $p>0$, and the $p$ th moment Lyapunov exponent should not be greater than $-\left(\lambda+4-\varepsilon_{2}\right)$.

Remark 22. The aim of the time-dependent term in our constructed stochastic Lyapunov function is to eliminate the effect of nonlocal kernel function.

\section{Conclusion}

In this paper, we have established the stochastic Lyapunov techniques for SDEs driven by $\mathrm{fBm}$. The obtained results are very effective to verify the two important types of stability, that is, stability in probability and moment exponential stability, for a given stochastic systems driven by $\mathrm{fBm}$. Also, it opens a possible way to stochastic stabilization and destabilization problem associated with SDEs driven by $\mathrm{fBm}$.

\section{Conflict of Interests}

The authors declare that there is no conflict of interests regarding the publication of this paper.

\section{Acknowledgments}

The authors would like to thank the anonymous referees for their valuable comments and suggestions. This work was partly supported by the National Natural Science Foundation of China (nos. 11301090, 11271139, and 61104138), the Fundamental Research Funds for the Central Universities (no. 2014ZB0033), and Science and Technology Planning Project of Tianhe District, Guangzhou (no. 201301YG027).

\section{References}

[1] A. N. Kolmogorov, "The Wiener spiral and some other interesting curves in Hilbert space," Doklady Akademii Nauk SSSR, vol. 26, pp. 115-118, 1940.

[2] H. E. Hurst, "Long-term storage capacity in reservoirs," Transactions of the American Society of Civil Engineers, vol. 116, pp. 400-410, 1951.

[3] H. E. Hurst, "Methods of using long-term storage in reservoirs," Proceedings of the Institution of Civil Engineers Part I, chapter 5, pp. 519-590, 1956. 
[4] B. B. Mandelbrot and J. W. van Ness, "Fractional Brownian motions, fractional noises and applications," SIAM Review, vol. 10, pp. 422-437, 1968.

[5] L. Decreusefond and A. S. Üstünel, "Fractional brownian motion: theory and applications," ESAIM Proceedings, vol. 5, pp. 75-86, 1998.

[6] F. Biagini, Y. Hu, B. Øksendal, and T. Zhang, Stochastic Calculus for Fractional Brownian Motion and Applications, Springer, Berlin, Germany, 2008.

[7] Y. S. Mishura, Stochastic Calculus for Fractional Brownian Motion and Related Processes, vol. 1929, Springer, Berlin, Germany, 2008.

[8] L. C. G. Rogers, "Arbitrage with fractional Brownian motion," Mathematical Finance, vol. 7, no. 1, pp. 95-105, 1997.

[9] C. Dellacherie and P. A. Meyer, Probabilities and Potential B, vol. 72, North Holland Publishing, Amsterdam, The Netherlands, 1982.

[10] L. Decreusefond and A. S. Üstünel, "Stochastic analysis of the fractional Brownian motion," Potential Analysis, vol. 10, no. 2, pp. 177-214, 1999.

[11] S. J. Lin, "Stochastic analysis of fractional Brownian motions," Stochastics and Stochastics Reports, vol. 55, no. 1-2, pp. 121-140, 1995.

[12] W. Dai and C. C. Heyde, "Itô's formula with respect to fractional Brownian motion and its application," Journal of Applied Mathematics and Stochastic Analysis, vol. 9, no. 4, pp. 439-448, 1996.

[13] T. E. Duncan, Y. Hu, and B. Pasik-Duncan, "Stochastic calculus for fractional Brownian motion. I. Theory," SIAM Journal on Control and Optimization, vol. 38, no. 2, pp. 582-612, 2000.

[14] E. Alòs, O. Mazet, and D. Nualart, "Stochastic calculus with respect to Gaussian processes," The Annals of Probability, vol. 29, no. 2, pp. 766-801, 2001.

[15] P. Carmona, L. Coutin, and G. Montseny, "Stochastic integration with respect to fractional Brownian motion," Annales de l'Institut Henri Poincare (B) Probability and Statistics, vol. 39, no. 1, pp. 27-68, 2003.

[16] R. J. Elliott and J. van der Hoek, "A general fractional white noise theory and applications to finance," Mathematical Finance, vol. 13, no. 2, pp. 301-330, 2003.

[17] F. Biagini, B. Øksendal, A. Sulem, and N. Wallner, "An introduction to white-noise theory and Malliavin calculus for fractional Brownian motion," Proceedings of The Royal Society of London A, vol. 460, no. 2041, pp. 347-372, 2004.

[18] M. Jolis, "On the Wiener integral with respect to the fractional Brownian motion on an interval," Journal of Mathematical Analysis and Applications, vol. 330, no. 2, pp. 1115-1127, 2007.

[19] E. Alòs and D. Nualart, "Stochastic integration with respect to the fractional Brownian motion," Stochastics and Stochastics Reports, vol. 75, no. 3, pp. 129-152, 2003.

[20] M. Gradinaru, I. Nourdin, F. Russo, and P. Vallois, “ $m$-order integrals and generalized Itô's formula: the case of a fractional Brownian motion with any Hurst index," Annales de l'Institut Henri Poincare (B) Probability and Statistics, vol. 41, no. 4, pp. 781-806, 2005.

[21] C. Bender, "An Itô formula for generalized functionals of a fractional Brownian motion with arbitrary Hurst parameter," Stochastic Processes and Their Applications, vol. 104, no. 1, pp. 81-106, 2003.

[22] C. Zeng, Q. Yang, and Y. Q. Chen, "Solving nonlinear stochastic differential equations with fractional Brownian motion using reducibility approach," Nonlinear Dynamics, vol. 67, no. 4, pp. 2719-2726, 2012.
[23] C. Zeng, Y. Chen, and Q. Yang, "Almost sure and moment stability properties of fractional order Black-Scholes model," Fractional Calculus and Applied Analysis, vol. 16, no. 2, pp. 317331, 2013.

[24] X. Mao, Stochastic Differential Equations and Their Applications, Horwood Publishing, Chichester, UK, 1997.

[25] C. Zeng, Y. Chen, and Q. Yang, “The fBm-driven OrnsteinUhlenbeck process: probability density function and anomalous diffusion," Fractional Calculus and Applied Analysis, vol. 15, no. 3, pp. 479-492, 2012. 


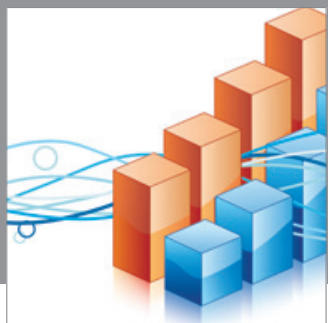

Advances in

Operations Research

mansans

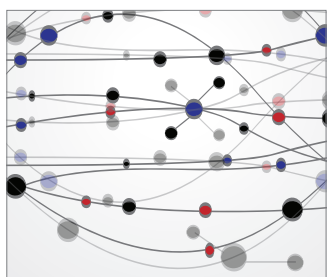

The Scientific World Journal
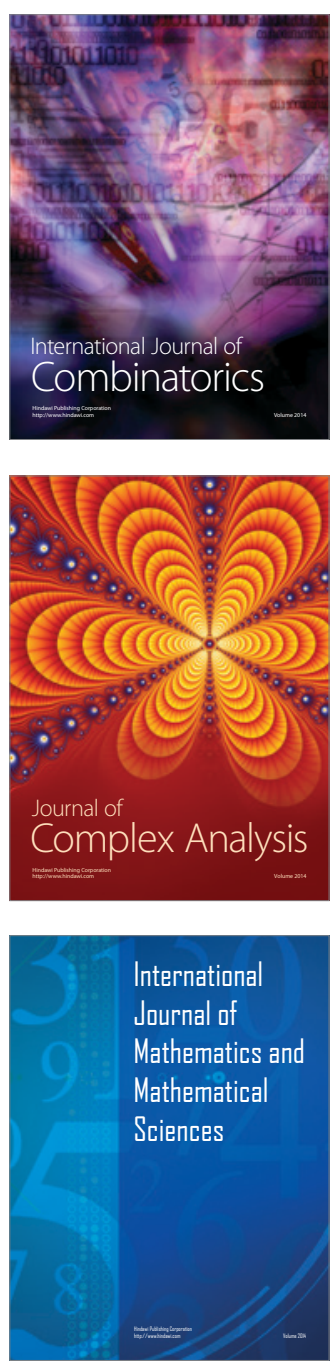
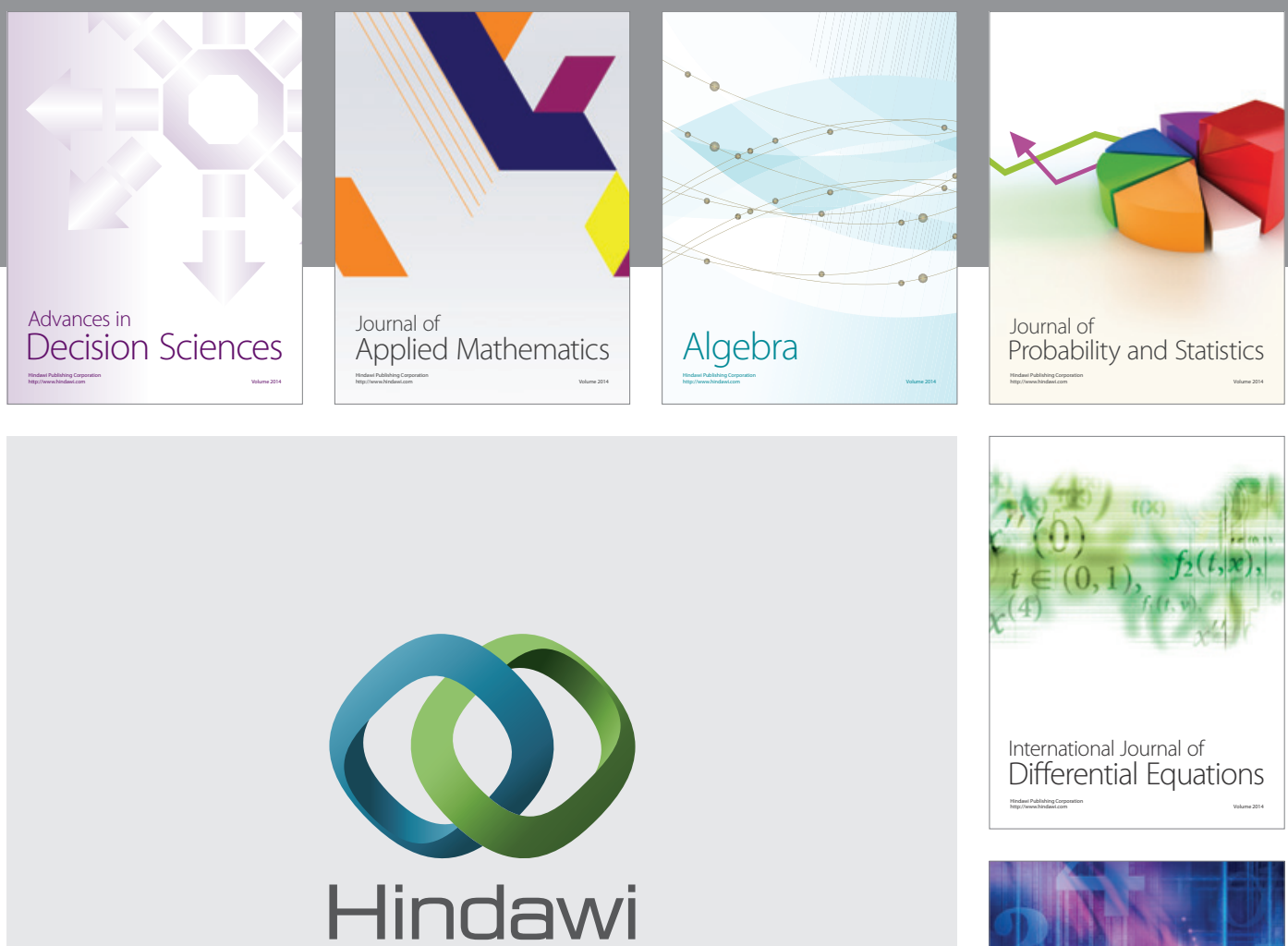

Submit your manuscripts at http://www.hindawi.com
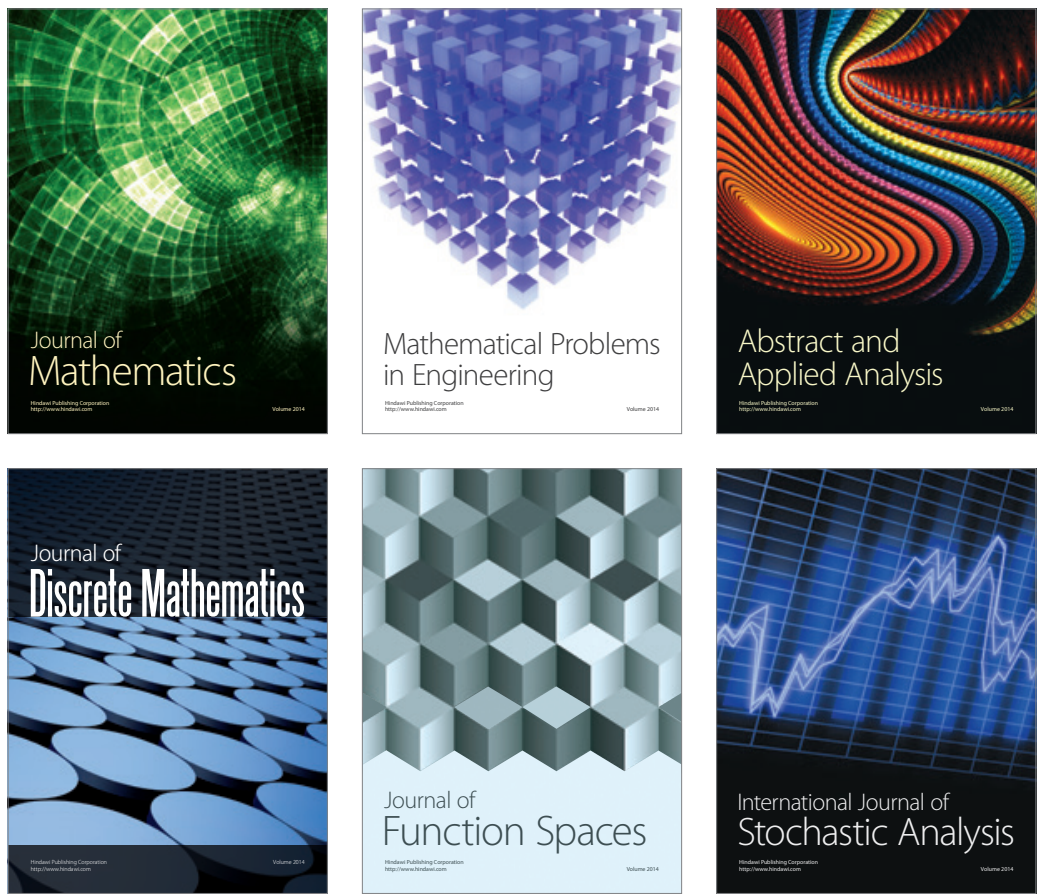

Journal of

Function Spaces

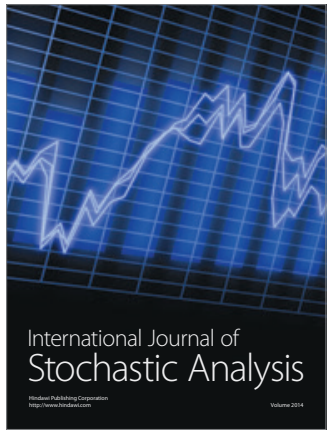

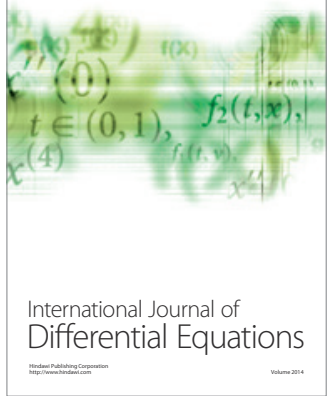
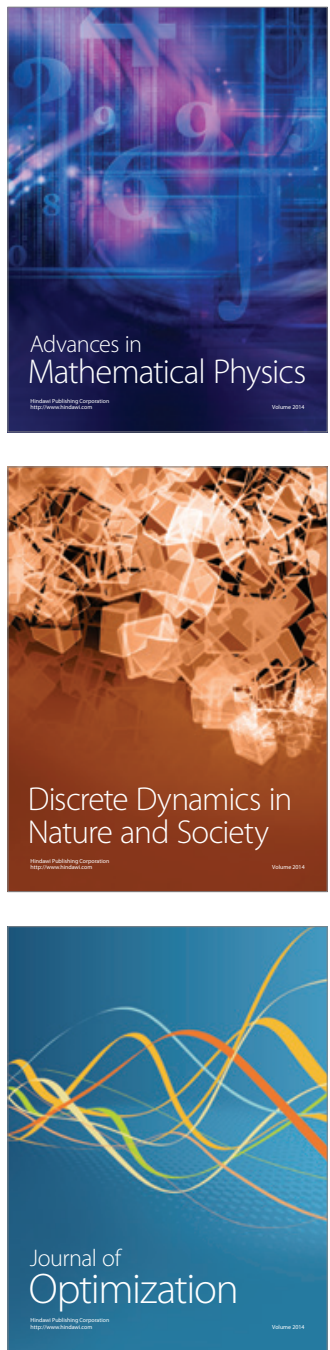